\title{
Diabetesdiagnose: Eine Messung reicht nicht
}

\author{
Zur Diagnose eines Diabetes empfiehlt die Deutsche \\ Diabetes-Gesellschaft (DDG), $\mathrm{HbA}_{1 c}$ und Blutglukose \\ zu bestimmen. Ein Experte hält dieses Vorgehen für \\ vernünftig und plädiert für einheitliche Diagnostik.
}

Zur Diabetesdiagnose sind nach den aktuellen Leitlinien der amerikanischen Diabetesgesellschaft (ADA) 4 Grenzwerte gleichwertig: Nüchtern-Plasmaglukose ( $\geq 126 \mathrm{mg} / \mathrm{dl}, \mathrm{PG}), 2-$ Stunden-PG ( $\geq 200 \mathrm{mg} / \mathrm{dl}$ ) im oralen Glukosetoleranztest (oGGT), der $\mathrm{HbA}_{1 \mathrm{c}}$ ( $\geq 6,5 \%$ ) und bei klassischen Symptomen der Hyperglykämie oder hyperglykämischer Krise die Zufallsglukose ( $\geq 200 \mathrm{mg} / \mathrm{dl}$ ).

\section{$\mathrm{HbA}_{1 \mathrm{c}}$ filtert andere „Diabetiker" als Glukosewert?}

„Das Problem an einer solchen Gleichstellung der Grenzwerten von $\mathrm{HbA}_{1 \mathrm{c}}$ und Glukosewerten ist, dass wir dadurch womöglich ganz unterschiedliche Populationen identifizieren“, machte PD Dr. Wolfgang Rathmann vom Deutschen Diabeteszentrum in Düsseldorf beim DiabetesUpdate aufmerksam. Im Normalfall würden anhand der glukosebasierten Kriterien weitaus mehr Personen mit Diabetes identifiziert, nämlich etwa ein Drittel mehr, als mit dem $\mathrm{HbA}_{1 \mathrm{c}}$. Sprich, es gibt viele Patienten, die erhöhte Glukose-, aber normale $\mathrm{HbA}_{1 \mathrm{c}}$-Werte haben und auch ein paar mit erhöhten $\mathrm{HbA}_{1 c^{-}}$, aber normalen Glukosewerten. $\mathrm{Ob}$ diese beiden

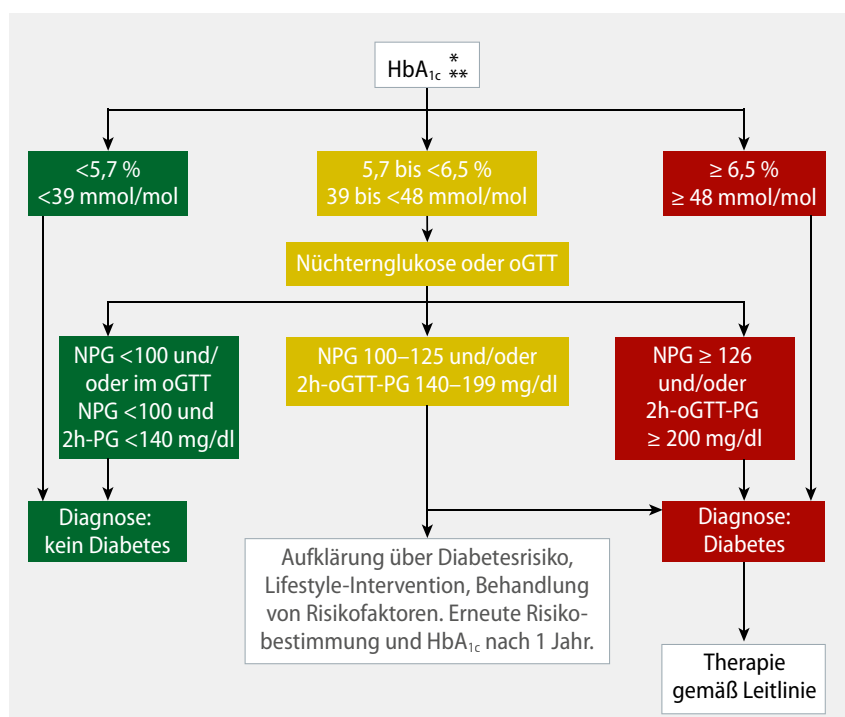

*bei Diabetes-Symptomen zusätzlich sofortige Glukosemessung

*** wenn eine Verfälschung des $\mathrm{HbA}_{\mathrm{tc}}$-Wertes zu erwarten ist, primär Diagnose durch Glukosemessung $\mathrm{NPG}=$ Nüchtern-Plasmaglukose, $2 \mathrm{~h}$-oGTT-PG; $2 \mathrm{~h}$-Plasmaglukose im oralen Glukosetoleranztest $(75 \mathrm{~g})$

1

Diagnostik laut DDG-Praxisempfehlung bei Diabetessymptomen u./o. -Risiko: Bei Wert deutlich $>/<$ angegebener/m Glukose/HbA und klinischer Plausibilität reicht meist Einzeltest. Grundsätzlich ist eine zeitnahe/parallele Bestätigung durch Test des gleichen oder jeweils anderen Parameters wünschen, v.a. in der Nähe der Grenzwerte; Pflicht ist dies bei kleinerer Abweichung als die Minimal

Difference (Weiteres s. Originaltext DDG).
Populationen mit neu entdecktem Diabetes dieselbe Prognose und Lebenserwartung haben, sei in Studien bisher noch gar nicht untersucht worden, bemerkte der Diabetologe kritisch. Auch wenn man natürlich vermuten könne, dass bei den Personen mit normalem $\mathrm{HbA}_{1 \mathrm{c}}$, aber erhöhter Glukose, das $\mathrm{HbA}_{1 \mathrm{c}}$ im Verlauf weiter ansteige. Die DDG hat sich daher in ihrem 2016 aktualisierten Praxisleitfanden [1] dafür entschieden, eine Kombination beider Messmethoden - also die Bestimmung des $\mathrm{HbA}_{1 \mathrm{c}}$ und eine glukosebasierte Messung - zur Diagnose eines Diabetes zu empfehlen. Gerade wenn das $\mathrm{HbA}_{1 \mathrm{c}}$ im prädiabetischen Grenzbereich - also bei 5,7 bis 6,5 - liegt, wird auf die Bedeutung einer zusätzlichen Messung der Nüchternglukose oder eines oGGT hingewiesen. Rathman hält diese Vorgehensweise für vernünftig. Denn neben den unterschiedlichen Populationen, die man womöglich durch nur eine Messung identifiziere, gebe es viele Störfaktoren, die den $\mathrm{HbA}_{1 c}$-Wert beeinflussen könnten. Dazu gehören Alter $\left(\mathrm{HbA}_{1 \mathrm{c}}\right.$ steigt mit Alter an) und Ethnizität (z. B. haben Afroamerikaner höhere Werte als Kaukasier), Anämien und Hömoglobinopathien, Schwangerschaft und Urämie. Wenn eine solche Verfälschung zu erwarten ist, sollte man gemäß dem Praxisleitfaden sofort auf eine glukosebasierte Messung zurückgreifen.

Rathman glaubt aber nicht, „dass die DDG es bisher nicht geschafft hat, diese Richtlinien in der Praxis durchzusetzen“. „Meine persönliche Beobachtung aus dem Bekannten- und Verwandtenkreis ist, dass der Diabetes in Deutschland sehr heterogen diagnostiziert wird“, sagte er. Hausärzte etwa würden sehr unterschiedlich vorgehen. „Das ist kein Vorwurf“, so der Diabetologe. Seiner Ansicht nach sollte die DDG daher aktiv kommunizieren, dass die Diabetesdiagnose ein einheitliches Vorgehen verlangt.

\section{Was tun bei zwei unterschiedlichen Testergebnissen?}

Wie aber sollte man vorgehen, wenn die Messwerte zweier Tests unterschiedlich ausfallen, also etwa der $\mathrm{HbA}_{1 \mathrm{c}}$ über dem Grenzwert liegt, die Nüchternglukose aber nicht? Solche Fälle kommen Rathman zufolge im Praxisalltag durchaus häufig vor.

Die ADA hat dazu in ihren im Januar 2017 aktualisierten Empfehlungen Stellung genommen [2]. Demnach sollte der Test, der über dem Grenzwert gelegen hatte, möglichst zeitnah - also etwa zwei bis drei Wochen später - wiederholt werden. Liegt dann das Ergebnis erneut über der Schwelle, ist die Diagnose gesichert. Generell empfiehlt die ADA, nach einem ersten, über dem Grenzwert liegenden Testergebnis denselben Test unter Verwendung einer neuen Blutprobe zeitnah zu wiederholen. Eine solche Sicherung der Diagnose sei in Anbetracht der Konsequenzen, die eine Diabetesdiagnose mit sich bringe etwa für das Abschließen einer Lebensversicherung, dringend nötig, wenn nicht entsprechende Symptome des Diabetes vorliegen, so Rathman. Veronika Schlimpert

\footnotetext{
Literatur:

1. Müller Wieland D et al. Definition, Klassifikation und Diagnostik des Diabetes Mellitus. Diabetologe 2016; 11(Suppl 2):78-81

2. ADA Classification and Diagnosis of Diabetes. Diabetes Care 2017; 40 (S 1):11
} 